\title{
Functional Identification of a Pulvinar Path from Superior Colliculus to Cortical Area MT
}

\author{
Rebecca A. Berman and Robert H. Wurtz \\ Laboratory of Sensorimotor Research, National Eye Institute, National Institutes of Health, Bethesda, Maryland 20982-4435
}

The idea of a second visual pathway, in which visual signals travel from brainstem to cortex via the pulvinar thalamus, has had considerable influence as an alternative to the primary geniculo-striate pathway. Existence of this second pathway in primates, however, is not well established. A major question centers on whether the pulvinar acts as a relay, particularly in the path from the superior colliculus (SC) to the motion area in middle temporal cortex (MT). We used physiological microstimulation to identify pulvinar neurons belonging to the path from SC to MT in the macaque. We made three salient observations. First, we identified many neurons in the visual pulvinar that received input from SC or projected to MT, as well as a largely separate set of neurons that received input from MT. Second, and more importantly, we identified a subset of neurons as relay neurons that both received SC input and projected to MT. The identification of these relay neurons demonstrates a continuous functional path from SC to MT through the pulvinar in primates. Third, we histologically localized a subset of SC-MT relay neurons to the subdivision of inferior pulvinar known to project densely to MT but also localized SC-MT relay neurons to an adjacent subdivision. This pattern indicates that the pulvinar pathway is not limited to a single anatomically defined region. These findings bring new perspective to the functional organization of the pulvinar and its role in conveying signals to the cerebral cortex.

\section{Introduction}

Visual neuroscientists have long recognized that the sense of sight emerges not only from processes within the retina and within the cerebral cortex, but also from the transformations that take place in the pathways linking the two. The primary path is well established: retinal signals are relayed through the lateral geniculate nucleus (LGN) of the thalamus to primary visual cortex (V1). For decades, however, a second path has been posited to provide an alternative route that bypasses LGN and V1 (Diamond and Hall, 1969). In this second path, retinal signals travel to the superficial layers of the superior colliculus (SC) in the brainstem and are likely relayed to cortex via a different thalamic nucleus, the pulvinar. The pulvinar is the largest nucleus that processes visual information (Jones, 2007), and it continues to be among the most perplexing. Much of what we know about the visual pulvinar comes from anatomical studies of its myriad subcortical and cortical connections (for review, see Grieve et al., 2000; Kaas and Lyon, 2007). While in many respects these connections suggest an orderly organization, a clear functional parcellation has yet to emerge. Physiological studies have identified pulvinar regions in which neurons respond to visual stimulation and can be modulated by attention and eye movements (for review, see Robinson,

Received Dec. 12, 2009; revised Jan. 25, 2010; accepted Feb. 16, 2010.

This work was supported by the National Eye Institute Intramural Research Program at the National Institutes of Health. We are grateful to Mitchell Smith for processing and analysis of the histological sections, to John McClurkin for assistance in modifying the computer programs to control microstimulation, and to Altah Nichols and Tom Ruffner for machine shop support.

Correspondence should be addressed to Rebecca A. Berman, Laboratory of Sensorimotor Research, National Eye Institute, Building 49, Room 2A50, 49 Convent Drive, Bethesda, MD 20982-4435. E-mail: bermanr@nei.nih.gov.

D01:10.1523/JNEUROSCI.6176-09.2010

Copyright $\odot 2010$ the authors $\quad 0270-6474 / 10 / 306342-13 \$ 15.00 / 0$
1993), but these findings have been difficult to relate directly to its structural subdivisions.

One organizing feature of the pulvinar may be the pathways that ascend through it to the cerebral cortex. The study of ascending pathways has proved invaluable for understanding another part of the thalamus, the medial dorsal nucleus. A subregion of this nucleus projects to a specific region of frontal cortex and conveys specific information for monitoring eye movements (Sommer and Wurtz, 2002). Knowledge of what the pulvinar conveys to specific cortical regions might likewise reveal critical aspects of its organization. The most intensively investigated path through the pulvinar originates in the superficial SC and is thought to project to the visual motion area of cortex, the middle temporal area (MT). This pathway has generated considerable interest as a source of visual signals in patients with blindsight, who are effectively blind after damage to V1 but nevertheless retain some visual abilities (Sanders et al., 1974). Surprisingly, however, there is no unequivocal evidence for the SC-pulvinar-MT path in primates, and recent anatomical studies have questioned the continuity of a pathway from SC to MT (Stepniewska et al., 2000).

In the present study, we used a novel combination of physiological techniques in awake macaque monkeys to determine whether a pulvinar path ascends from SC to MT (Fig. 1). We used electrical activation of synaptic input to the pulvinar from SC (orthodromic stimulation from SC) and backfiring of the pulvinar projection to MT (antidromic stimulation from MT) to identify neurons belonging to the ascending path. We then determined their location on the basis of physiological maps and subsequent histology. With this approach, we successfully identified and localized an ascending path from SC to MT in the primate pulvinar. 


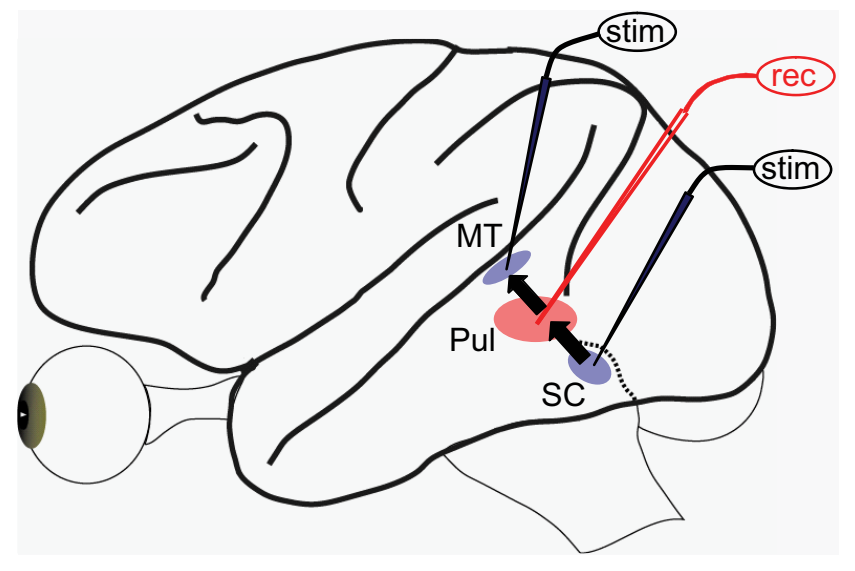

Figure 1. Combined microstimulation (stim) and recording (rec) were used to identify single pulvinar neurons that belong to the ascending pathway from SC to pulvinar to MT. A microelectrode recorded spikes of a single pulvinar neuron. Stimulating microelectrodes activated neurons in the lower superficial layers of $\mathrm{SC}$ and in cortical area MT. This procedure established the connectivity of the pulvinar neuron on the basis of its orthodromic (synaptic) or antidromic (backfired) activation from SC and MT.

\section{Materials and Methods}

Experiments were conducted in three male macaque monkeys (designated YZ, AM, and OM), weighing 8-12 kg. Monkeys were implanted with scleral search coils for measuring eye position and with an acrylic base to accommodate recording chambers and a post for immobilizing the head during experiments (for details, see Sommer and Wurtz, 2000). All procedures were approved by the Institute Animal Care and Use Committee and complied with Public Health Service policy on the humane care and use of laboratory animals.

Behavioral tasks. Visual stimuli were back-projected by a DPI projector onto a tangent screen located $57 \mathrm{~cm}$ in front of the monkey. A computer running REX (Hays et al., 1982) controlled stimulus presentation, reward administration, the recording of eye movements and neuronal activity, and the online display of results. We used a simple fixation task to map receptive fields in the pulvinar, SC, and MT. The monkey began each trial by fixating a small central spot $\left(0.4^{\circ}\right)$ for an initial period of $100-500 \mathrm{~ms}$, after which a visual stimulus appeared for an additional $500-1000 \mathrm{~ms}$. The trial ended when the fixation spot disappeared $250 \mathrm{~ms}$ later and the monkey received liquid reward for maintaining fixation throughout the trial within a $\pm 2^{\circ}$ electronic window. We used a delayed visually guided saccade task to assess visual and presaccadic activity. Trials began with an initial fixation of 100-500 ms. A target (typically a $1^{\circ}$ spot) was then presented in the periphery, but the monkey had to continue fixating for an additional 500-1000 ms until the fixation spot disappeared, cueing the monkey to make a saccade to the target. Liquid reward was given if the monkey attained the target within $500 \mathrm{~ms}$ and maintained fixation there for an additional $500 \mathrm{~ms}$. For area MT, we mapped receptive fields and determined directional tuning using random dot patterns, sine wave stimuli, or bar stimuli moving with a variety of speeds and directions. For the pulvinar neurons, stimuli were typically random dot patterns or spots of varying size, but we sometimes used bar stimuli and sine-wave gratings or had the monkey make delayed visually guided saccades. We also used simple experimenter-generated auditory stimuli (e.g., hand-clapping) to identify the medial geniculate nucleus, which proved a useful landmark for the inferior pulvinar.

Recording chambers. We recorded from single neurons in the pulvinar and microstimulated in SC and area MT with tungsten microelectrodes (Frederick Haer) advanced by a stepper microdrive. Electrodes passed through guide tubes that were placed in a $1-\mathrm{mm}$-resolution grid in the recording chamber (Crist et al., 1988). We accessed the pulvinar and area MT through the same recording chamber. In monkey YZ, we used a single chamber for each hemisphere, placed at stereotaxic coordinates A6 L12. For monkeys AM and OM, a single rectangular chamber allowed access to pulvinar and MT in both hemispheres and was centered on the midline at A4. For all three monkeys, we accessed SC through a single cylindrical chamber, tilted $38^{\circ}$ top backward from vertical so that electrodes were directed anteriorly and approached the SC approximately normal to its surface.

Localization of the pulvinar. Our recordings targeted the lateral (PL) and inferior (PI) divisions of the pulvinar, which contain visually responsive neurons and are considered the most likely candidates for relay neurons (Bender, 1981; Petersen et al., 1985; Cusick et al., 1993; Stepniewska et al., 2000; Lyon et al., 2005). We also recorded from the medial pulvinar (PM), particularly near its border with PL, where Petersen et al. (1985) identified a visually responsive zone they called Pdm. PI and PL occupy the posterior part of the pulvinar, with PM occupying more anterior and medial regions. Much of the visual pulvinar has been further subdivided on the basis of architectonic and immunohistochemical markers (for review, see Kaas and Lyon, 2007). These subdivisions have been described as "nuclei" or "subnuclei," and the terms sometimes connote differing hypotheses regarding the organization of the pulvinar (e.g., Gutierrez et al., 1995). For simplicity, we chose the more neutral term "subdivision" and adopted the nomenclature of Stepniewska and Kaas (1997) and Adams et al. (2000) to describe these subdivisions. We used the LGN to guide us to areas of interest in the visual pulvinar because the pulvinar itself contains few clear physiological landmarks. In our initial recording, we searched for LGN on the basis of a structural MRI. LGN neurons were identifiable by the short-latency monocular visual responses and the signature alternation of ocularity as the electrode progressed through layers. Once we encountered LGN neurons, we determined our location on the LGN map based on the visual representations (Malpeli and Baker, 1975). We then targeted the posterior pole of the LGN, which typically demarcated the anterior and lateral borders of the pulvinar area of interest, and proceeded to map the pulvinar.

Stimulation electrodes. Stimulating electrodes in SC and MT were unipolar and of low impedance $(\sim 100 \mathrm{k} \Omega$ at $1 \mathrm{kHz})$. The low impedances greatly reduced stimulation artifact and extended the longevity of the electrodes, yet were still sufficient for determining the physiological properties of MT and SC neurons. We placed stimulating electrodes with the guidance of an initial MRI and subsequent neuronal recording to map the visual representations in each structure.

For MT, our electrodes approached vertically and so encountered MST first. Both MT and MST contain neurons tuned for directional motion (for review, see Maunsell and Newsome, 1987). Therefore we distinguished MT from MST by three criteria: (1) restricted contralateral receptive fields that scaled with eccentricity, (2) surround suppression, such that responses were strongest for small stimuli presented within the receptive field boundaries, and (3) a predictable progression of receptive field locations as the electrode descended. Histology from monkey YZ confirmed that electrode tracks were located in the densely myelinated zone known to correspond to physiological MT (Van Essen et al., 1981). We often placed two stimulating electrodes in MT, using separate guide tubes placed in adjacent grid holes. In some experiments we used only one MT stimulating electrode, or we inserted two thin electrodes (100 $\mu \mathrm{m}$ diameter) glued together with a fixed distance between tips (e.g., $1 \mathrm{~mm}$ ) to access different spatial representations within a single MT penetration.

For SC, electrodes approached normal to its surface and entry into the SC was unmistakable because neurons in the superficial-most layers have robust responsivity to visual stimuli. We used the visually guided saccade task to map visual receptive fields and look for presaccadic activity, and we microstimulated to evoke saccades with a train of biphasic pulses $(0.25 \mathrm{~ms} /$ phase at $350 \mathrm{~Hz}$ for $70 \mathrm{~ms}$; note that this stimulation protocol differs from the single pulses used to identify connected neurons, described subsequently). Our target was the lower superficial layers, which are the major source of input to the pulvinar (Benevento and Fallon, 1975; Harting et al., 1980). Previous stimulation and anatomical studies in macaque indicate that this electrode placement likely activated retino-recipient neurons of the superficial SC (Schiller and Malpeli, 1977; Benevento and Standage, 1983) as well as superficial neurons that receive input from other sources (Fries, 1984; Baizer et al., 1991). As we advanced the electrode, we closely monitored the visual and presaccadic activity as well as the current threshold needed to evoke saccades. We used two criteria to 
Table 1. Summary of stimulation sites in SC and MT

\begin{tabular}{|c|c|c|c|c|c|c|c|c|c|}
\hline \multirow[b]{3}{*}{ Monkey } & \multirow[b]{3}{*}{ Hemisphere } & \multicolumn{8}{|c|}{$\begin{array}{l}\text { Spatial representation at each site in degrees of horizontal }(x) \text { and } \\
\text { vertical }(y) \text { visual angle }\end{array}$} \\
\hline & & \multicolumn{2}{|l|}{$\mathrm{SC} 1$} & \multicolumn{2}{|l|}{$\mathrm{SC} 2$} & \multicolumn{2}{|l|}{ MT1 } & \multicolumn{2}{|l|}{ MT2 } \\
\hline & & $x$ & $y$ & $x$ & $y$ & $x$ & $y$ & $x$ & $y$ \\
\hline YZ & $\mathrm{L}$ & 10 & -5 & 10 & -15 & 20 & -20 & & \\
\hline$Y Z$ & $\mathrm{~L}$ & 1 & 0 & 5 & 10 & 30 & 15 & & \\
\hline YZ & L & 5 & 0 & 20 & -5 & 20 & 5 & & \\
\hline YZ & $\mathrm{L}$ & 8 & -2 & & & 20 & -5 & & \\
\hline$Y Z$ & $\mathrm{R}$ & -5 & 0 & & & -10 & 1 & -5 & -7 \\
\hline YZ & $\mathrm{R}$ & -10 & 0 & & & -10 & 1 & -5 & -10 \\
\hline YZ & $\mathrm{R}$ & -20 & -10 & -10 & 10 & -8 & 12 & -7 & 6 \\
\hline$Y Z$ & $\mathrm{R}$ & -7 & -5 & -10 & 5 & -8 & 12 & -7 & 6 \\
\hline AM & $\mathrm{L}$ & 15 & 5 & & & 5 & 15 & & \\
\hline AM & L & 3 & 10 & & & 1 & 6 & & \\
\hline AM & $\mathrm{R}$ & -20 & 5 & & & -20 & 10 & & \\
\hline AM & R & -13 & -2 & & & -15 & -5 & & \\
\hline AM & R & -8 & -1 & -5 & -8 & -12 & -4 & -6 & -12 \\
\hline AM & R & -10 & 0 & -5 & -10 & -12 & -4 & -6 & -12 \\
\hline $0 M$ & $\mathrm{R}$ & -5 & 20 & -4 & 1 & -5 & -15 & -5 & 3 \\
\hline OM & $\mathrm{R}$ & -6 & 10 & -5 & 1 & -6 & 13 & & \\
\hline
\end{tabular}

L, Left; $R$, right.

determine that we were in the lower superficial layers: (1) neuronal activity was strongly visual, and (2) the current to evoke saccades was greater than the $50 \mu \mathrm{A}$ indicative of the intermediate layers, usually between 100 and $150 \mu \mathrm{A}$.

We typically placed two stimulating electrodes in the SC to activate distinct spatial representations within the superficial SC. In a small number of early experiments we placed one electrode in superficial SC and another in the intermediate layers of SC (defined by robust presaccadic activity and $\leq 50 \mu \mathrm{A}$ to evoke saccades), or used a moveable electrode in SC. Only a handful of pulvinar neurons were activated from the intermediate SC $(n=3)$, consistent with a negligible anatomical projection from intermediate layers to the inferior and lateral pulvinar (Benevento and Fallon, 1975; Harting et al., 1980). These few neurons are not included here, and subsequently we placed stimulating electrodes only in the superficial SC. Once we determined that the stimulating electrodes were in the desired location, we used epoxy to fasten the electrode to the guide tube and the guide tube to the grid. We then recorded (and in the case of SC, also microstimulated) to verify their location. Stimulating electrodes were semichronic, staying in place up to 8 weeks at a time without incident. At the stimulation sites from which we activated pulvinar neurons, the mean spatial representations were $10.7^{\circ}$ in amplitude at an elevation of $31.7^{\circ}$ for SC, and $15.5^{\circ}$ in amplitude at an elevation of $42.6^{\circ}$ for MT. Table 1 summarizes the SC and MT stimulation sites for each monkey and hemisphere. As detailed in the following section, we rarely observed the activation of a single pulvinar neuron from both of the stimulation sites within SC or within MT. This observation indicates that the stimulating current led to activation of circumscribed spatial representations.

Identifying connected cells: orthodromic and antidromic stimulation. We used microstimulation to determine whether single pulvinar neurons were connected to SC and/or MT (for review, see Lemon, 1984). To identify a connected pulvinar neuron, we stimulated the SC and MT using single biphasic current pulses $(0.15 \mathrm{~ms} /$ phase, negative-positive) and looked for activation of the pulvinar neuron. If the recorded pulvinar neuron receives input from SC, then stimulation of SC can synaptically activate the pulvinar neuron. In this case, SC stimulation causes an action potential to be propagated in the normal forward-going, orthodromic direction along the axon, which drives the pulvinar neuron through the synapse. Variability in synaptic transmission causes the orthodromically driven action potential to appear as a jittery-latency spike in the pulvinar (variability $>0.1 \mathrm{~ms}$ ), as seen in the example neuron in Figure $2 \mathrm{~A}$. Furthermore, as shown in Figure $2 B$, orthodromic activation fails the collision test, which is described in detail for Figure $2 \mathrm{D}$. If the recorded pulvinar neuron sends output to MT, then stimulation of MT sends an
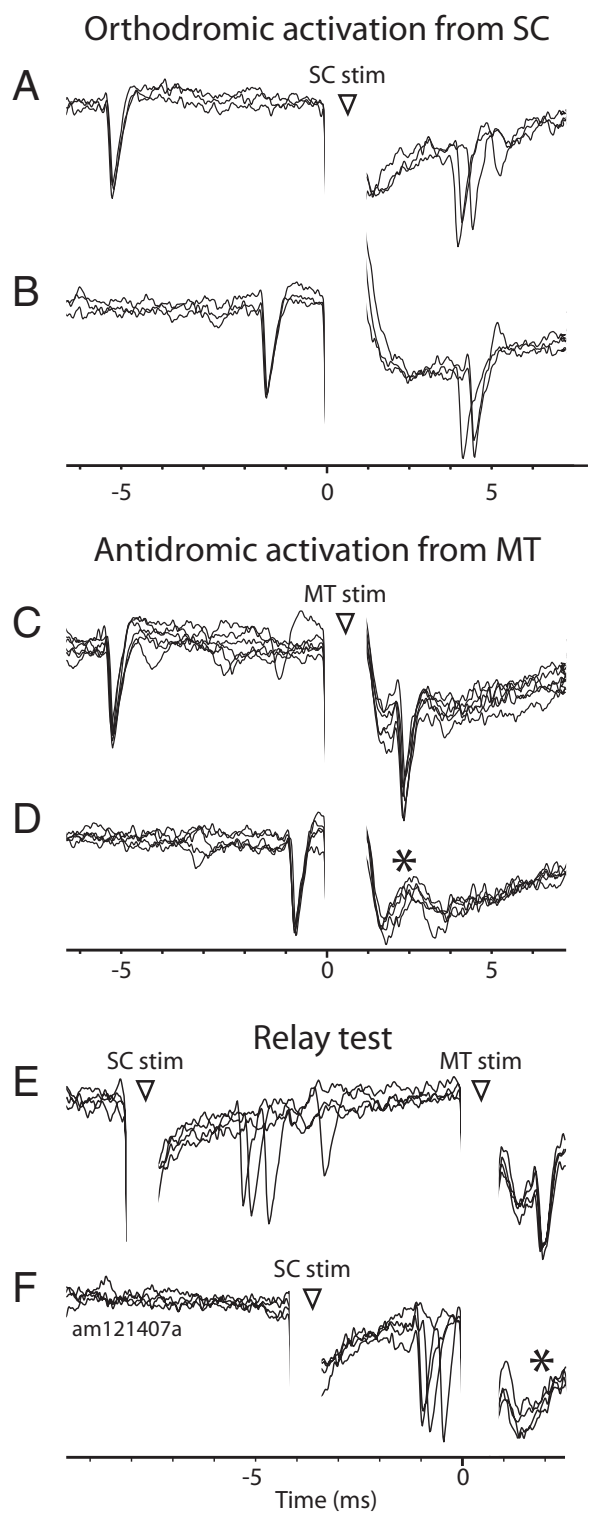

Figure 2. Example recording traces from a single pulvinar neuron show that it both receives input from SC and projects to MT, as evidenced by spikes evoked by stimulation of SC and MT. Each panel shows several stimulation trials superimposed; stimulus artifacts are erased for clarity. Time 0 indicates the start of stimulation (MT stimulation for the relay test). $A$, Orthodromic activation of the pulvinar cell indicates input from SC. Stimulation of SC causes the pulvinar neuron to fire a spike at a variable latency, $\sim 3 \mathrm{~ms}$ after stimulation. $\boldsymbol{B}$, The orthodromic spike fails to collide with a spontaneous spike that precedes stimulation at short latency. C, Antidromic activation indicates output to MT. Stimulation of MT elicits a spike from the pulvinar neuron at a fixed latency ( $\sim 1.7 \mathrm{~ms})$, consistent with backfiring the neuron. $\boldsymbol{D}$, The collision test confirms antidromic activation. MT stimulation is triggered immediately after a spontaneous spike in the pulvinar, which collides with the backfired spike (asterisk denotes its absence on collision trials). This is in contrast to $\boldsymbol{C}$, in which the triggering pulvinar spike occurs well before MT stimulation and therefore does not interfere with the antidromically evoked spike. $\boldsymbol{E}, \boldsymbol{F}$, The relay test demonstrates unequivocally that $\mathrm{SC}$ and $\mathrm{MT}$ stimulation activate the same pulvinar neuron. Here, MT stimulation follows a spike elicited by SC stimulation. $\boldsymbol{E}$, Long delay between $\mathrm{SC}$ and MT stimulation does not cause collision, and the MT-evoked spike is observed. $\boldsymbol{F}$, Short delay between SC and MT stimulation. The SC-evoked spike occurs just before MT stimulation and therefore collides the MT-evoked spike.

impulse in the backward, antidromic direction along the axon, which appears as a fixed-latency spike in the recorded pulvinar neuron (variability $<0.1 \mathrm{~ms}$ ) (Fig. $2 C$ ). The collision test verifies that the pulvinar neuron was antidromically activated. In this test, we trigger stimulation on a spontaneous spike in the pulvinar. When MT stimulation is trig- 
gered almost immediately after the spontaneous pulvinar spike (Fig. $2 D$ ), the spontaneous action potential travels up the axon as the antidromically driven impulse travels down, and the two cancel each other out or "collide." Consequently, the stimulation-evoked spike in the pulvinar is absent in Figure 2D. By contrast, the stimulation-evoked spike is present in Figure $2 C$ because there is a long $(5 \mathrm{~ms})$ delay between the spontaneous spike and MT stimulation. In this case, the spontaneous spike is outside of the collision window, i.e., it has traveled up the axon before MT stimulation backfires the neuron.

A further critical requirement for the identification of SC-MT relay neurons is that the very same neuron is both orthodromically activated from SC and antidromically activated from MT. Recording must be from the same pulvinar neuron during the orthodromic and antidromic tests rather than unintentionally from two simultaneously recorded neurons. We emphasize this distinction because it is possible to record simultaneously from two separate neurons, one orthodromically activated from SC and the other antidromically activated from MT. In fact, we recorded from four such colocalized pairs. For these cases, we were able to determine the presence of two different cells using two main criteria-by evaluating the spike waveforms and, often, by noting that one of the two activated spikes disappeared while the other remained. These colocalized pairs, though they indicated a region of proximal SC input and MT output, did not constitute relay neurons. By contrast, SC-MT relay neurons were clearly isolated single units with both orthodromic SC activation and antidromic MT activation, and we determined this using the same criteria- by evaluating the spike waveform and, often, by observing the clear simultaneous disappearance of this single neuron and its associated SC- and MT-evoked spikes. If time permitted, we ran a more definitive test, the relay test, to confirm that we were activating the same neuron from both SC and MT. The relay test is essentially an extension of the collision test (Zhu and Lo, 1998; Sommer and Wurtz, 2004). In the relay test, however, we do not use a spontaneous pulvinar spike to trigger MT stimulation. Instead we actively elicit the pulvinar spike with SC stimulation and arrange the timing so that the SC-evoked (orthodromic) spike precedes MT stimulation by either a long latency ( $8 \mathrm{~ms}$ ) (Fig. $2 E$ ) or a short latency $(4 \mathrm{~ms})$ (Fig. 2 F). At short latencies, if the SC-evoked spike collides with the MT-evoked spike and abolishes it, the relay test is successful and shows unequivocally that a single neuron both receives input from SC and projects to MT (Fig. $2 F$ ).

Stimulation of SC and MT can also reveal two other kinds of connectivity of single pulvinar neurons. First, SC stimulation can elicit an antidromically driven spike, revealing a pulvinar neuron that sends output to SC. This category was exceedingly rare $(n=2)$ and is not considered here. Second, MT stimulation can cause an orthodromically driven spike, revealing a pulvinar neuron that receives input from MT. We encountered these neurons more commonly and describe their localization. We did not keep counts of the many pulvinar neurons encountered (likely $>1000$ ) for which we were unable to identify a connection to either SC or MT.

A key consideration in the identification of connected neurons is the alignment of spatial representations in the stimulation sites and recording sites (Movshon and Newsome, 1996; Sommer and Wurtz, 2004). In our experiments, alignment is an issue at three levels: between the stimulation sites (SC-MT) and between each of these sites and the pulvinar (SC-pulvinar, MT-pulvinar). Obviously, spatial alignment of the SC-MT stimulation sites was determined by the placement the stimulating electrodes and accordingly we attended closely to the relationship between the receptive field representations at these two sites. With the exception of the first hemisphere, in which we concentrated on mapping the visual pulvinar and not on the alignment of stimulation electrodes (YZ-L in Table 1), we always endeavored to match the centers of SC and MT receptive fields within $5^{\circ}$ of one another. For the next four hemispheres for which we attempted this, the distance between the receptive-field centers of matched SC-MT pairs ranged from $1^{\circ}$ to $14.1^{\circ}$, with a median of $4.1^{\circ}$. We therefore had some control over the SC-MT alignment. By contrast, alignment between each of these stimulation sites and the pulvinar was determined by the receptive field of the neuron encountered during recording, which was often difficult to predict. For neurons for which we identified an input from SC and were able to map the pulvinar receptive field, the median distance between the SC-pulvi- nar representations was $7.4^{\circ}$, with a range of $0^{\circ}$ to $38.6^{\circ}$. The median distance between MT-pulvinar representations was larger: $12.8^{\circ}$ for neurons with identified output to $\mathrm{MT}\left(\right.$ range $0^{\circ}-39.1^{\circ}$ ) and $20.6^{\circ}$ for neurons with identified input from MT (range $3.6^{\circ}-44.7^{\circ}$ ). During recording, it was often our impression that alignment was critical for observing a connection, but in analysis of the total sample we were unable to detect an obvious, consistent relationship between alignment and either the likelihood of activation from a given stimulating electrode or the threshold required to identify a connected neuron.

We used the voltage drop across a series resistor in the stimulation circuit, measured with a high-impedance differential probe, to determine the minimum current needed to produce either orthodromic or antidromic activation of pulvinar neurons. Thresholds ranged from 100 to $2000 \mu \mathrm{A}$; we did not determine the precise value of currents less than $100 \mu \mathrm{A}$. Median thresholds were $500 \mu \mathrm{A}$ for SC input neurons, $600 \mu \mathrm{A}$ for MT output neurons, and $600 \mu \mathrm{A}$ for MT input neurons. Our practical experience indicated that current spread from the site of stimulation was minimal. Specifically, when a pulvinar neuron was activated from one SC site, the neuron was rarely activated from the other SC site, despite the fact that the median distance between the two stimulating electrodes on the SC map was estimated at $1.6 \mathrm{~mm}$ (Ottes et al., 1986). In a small number of cases $(n=7)$, the pulvinar neuron could be activated from both sites but always required more current (median $400 \mu \mathrm{A}$ ) from one of the two sites. We observed a similar pattern for MT, finding either antidromic or orthodromic activation from both MT sites for only a small number of neurons, again with threshold differences between electrodes (antidromic, $n=5$, median $285 \mu \mathrm{A}$; orthodromic $n=7$, median $275 \mu \mathrm{A}$ ).

Histology. Coronal sections $(50 \mu \mathrm{m})$ from monkey YZ were processed histologically for both the pulvinar and area MT. For the pulvinar, sections were stained alternately for Nissl and calbindin. The calbindin stain followed the protocol used by Kaas and colleagues (Celio, 1990; Stepniewska and Kaas, 1997), with modifications based on the protocol of Saleem and Logothetis (2007). The Nissl sections allowed identification of pulvinar recording sites and electrolytic marks, and the calbindin stain allowed for the immunohistological demarcation of subdivisions, particularly within the inferior pulvinar. We aligned the adjacent Nissl and calbindin images using multiple common landmarks, including the location of electrolytic marks, white matter tracts, and vasculature, as well as the overall structural outline. Physiological maps from the recording sessions were then aligned according to the known depths of the electrolytic marks. For MT, coronal sections were stained alternately for Nissl and for myelin. The myelin stain followed a modified Gallyas protocol and provided visualization of the densely myelinated zone known to correspond to physiological area MT (Van Essen et al., 1981).

\section{Results}

The central result of this study is that we have identified single neurons in the primate pulvinar that are part of the ascending pathway from SC to MT. In five hemispheres, we physiologically identified a total of 313 pulvinar neurons connected to SC, MT, or both (Table 2). Of these, SC-MT relay neurons proved a small and nearly elusive fraction $(\sim 8 \%)$, but were nonetheless identifiable as single neurons that both received input from SC and sent output to MT. We describe these as SC-MT relay neurons to distinguish them from other kinds of relay neurons (e.g., corticocortical relays) located in the pulvinar, but for simplicity we

Table 2. Summary of identified pulvinar neurons with demonstrated connections to SC and MT

\begin{tabular}{lr}
\hline Type of neuron & \multicolumn{1}{c}{} \\
\hline Relay (input from SC and output to MT) & 24 \\
Input from SC, no other identified connection & 157 \\
Output to MT, no other identified connection & 64 \\
Input from MT, no other identified connection & 64 \\
Input from MT and input from SC & 2 \\
Output to SC & 2 \\
\hline
\end{tabular}


typically use the abbreviation "relay" to describe this class of neurons in the present paper. Of the remaining identified neurons, the majority fell into the two categories relevant to the ascending pathway: input from SC ( 50\%) and output to MT $(\sim 21 \%)$. Stimulation also allowed for the identification of two categories of neurons that do not belong to the ascending path but are of potential interest: pulvinar neurons that projected to SC, and pulvinar neurons that received input from MT. We found only two neurons that sent output to SC $(<1 \%)$ and they are not considered further. In contrast, we found many neurons that received input from MT ( 21\%), two of which also received input from SC, and these are included for examining the reciprocal interaction between pulvinar and cortex.

\section{Maps in the visual pulvinar}

Our first objective was to obtain maps of visual representations in the pulvinar in order to establish a framework for localizing the neurons connected to SC and MT. Specifically, we sought to determine the presence of retinotopic and nonretinotopic zones previously described in the inferior and lateral pulvinar (Bender, 1981; Ungerleider et al., 1984; Petersen et al., 1985; Adams et al., 2000; Shipp, 2001). These studies indicate that the retinotopic zone is comprised of two separate maps, which we did not seek to delineate in detail. Our primary intention was to detect the transition between a more lateral retinotopic zone and a more medial nonretinotopic zone, particularly because the nonretinotopic regions likely correspond to subdivisions with dense SC and MT connectivity (Lin and Kaas, 1979; Ungerleider et al., 1984; Adams et al., 2000; Stepniewska and Kaas, 2000; Shipp, 2001). In the first monkey (YZ), we characterized the receptive fields of all visual neurons encountered during recording. The resultant maps (Fig. 3) show the visual representations for the coronal sections through the pulvinar area of interest. For each hemisphere, we display the three sections in which we observed the greatest concentration of pulvinar cells connected to SC or MT, though the maps indicate all visual neurons regardless of connectivity. All sections from both hemispheres are shown with medial on the left and lateral on the right. We mapped the left hemisphere first (Fig. $3 A-C$ ). These coronal sections begin at the level of posterior LGN (section P0) and reveal the presence of two major zones. In the lateral region of the pulvinar, we found evidence for a retinotopic zone, which had been defined previously by contiguous but distinct regions representing the lower and upper visual fields (Bender, 1981). This zone is evident in Figure 3, $A$ to $C$, from the preponderance of
Monkey YZ
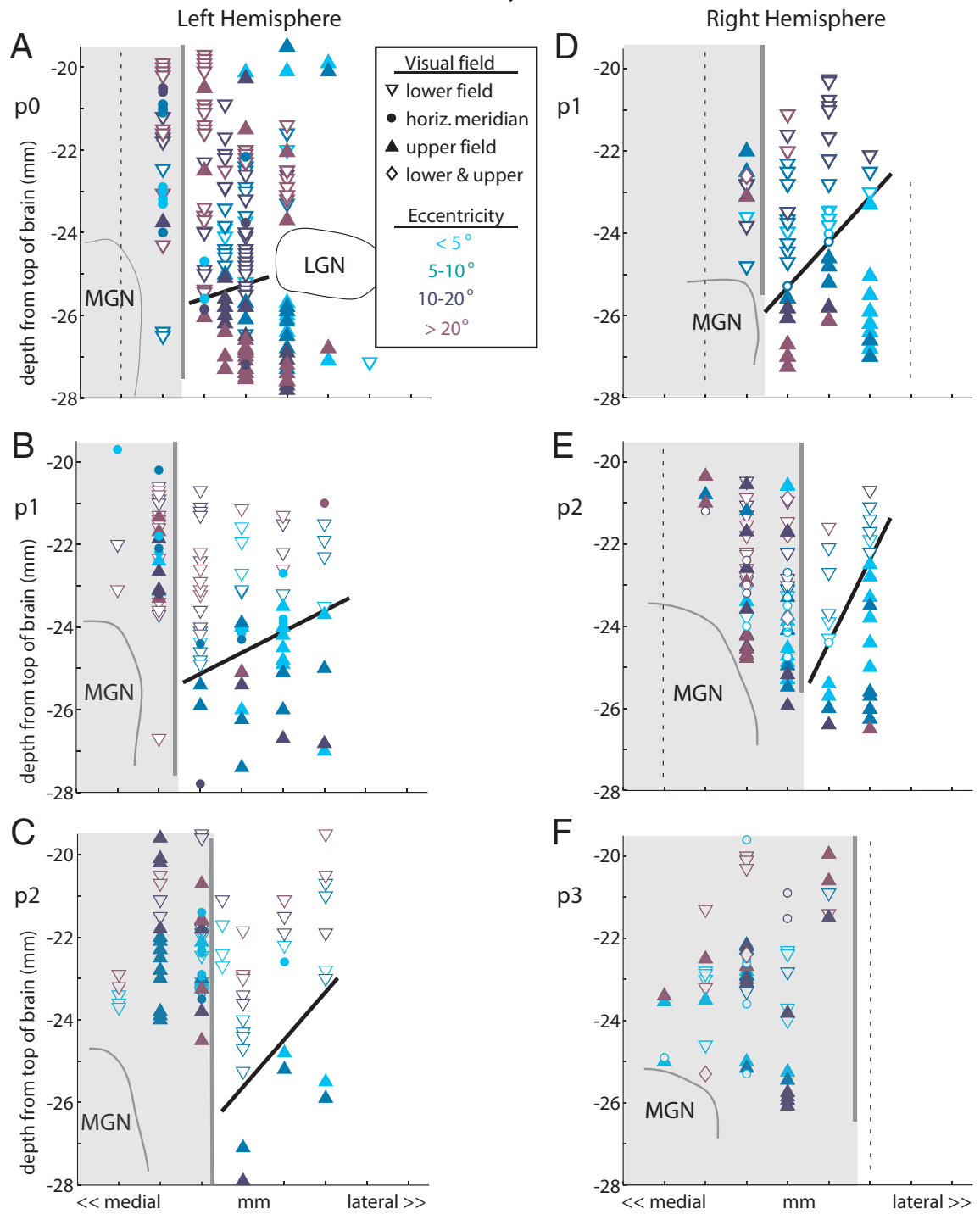

Figure 3. Visual field maps in the pulvinar indicate areas of retinotopic and nonretinotopic organization. The two columns show data from the left and right hemispheres of monkey $Y Z$, in which extensive field mapping was conducted. Panels show drawings of coronal sections through the area of interest for the relay pathway, and move in $1 \mathrm{~mm}$ intervals from anterior (top) to posterior (bottom).A-C, Data from the left hemisphere begin at the level of the posterior pole of LGN (section P0, top panel). D-F, Data from the right hemisphere begin just posterior to the LGN (section P1). Each panel shows the location in depth in mm ( $y$-axis) of visual neurons encountered at each medial-lateral site in the recording grid in $\mathrm{mm}$ ( $x$-axis). In this and subsequent figures, medial is on the right and lateral on the left of each panel, regardless of hemisphere. Symbols indicate the field representation of the neuron and colors indicate approximate eccentricity of the receptive field (see legend). The black line denotes the estimated transition from lower to upper visual field representations (horizontal meridian). The vertical gray line denotes the estimated border between a retinotopic zone, in which we could detect a systematic transition from lower field to upper field representations as the electrode descended, and a nonretinotopic zone, in which no systematic pattern was detected. For the left hemisphere, maps include a small number of neurons obtained $0.5 \mathrm{~mm}$ posterior to the displayed section (obtained with an offset grid). Some pulvinar cells with connections are not displayed because they were lost before receptive fields were mapped, or were not readily driven by visual stimulation. Vertical dotted lines indicate sites at which recording was done but no visual neurons were encountered.

downward-pointing symbols in the upper right part of the section, which indicate visual receptive fields whose centers were below the horizontal meridian. Representations of the horizontal meridian, shown with circles, separate these lower field maps from the upper field maps, indicated by the upward-pointing symbols in the lower right part of the section. In contrast, more medially in the pulvinar (Fig. 3A-C, leftward portions of each section) we found a nonretinotopic zone with no systematic progression of visual field representations. This zone is consis- 

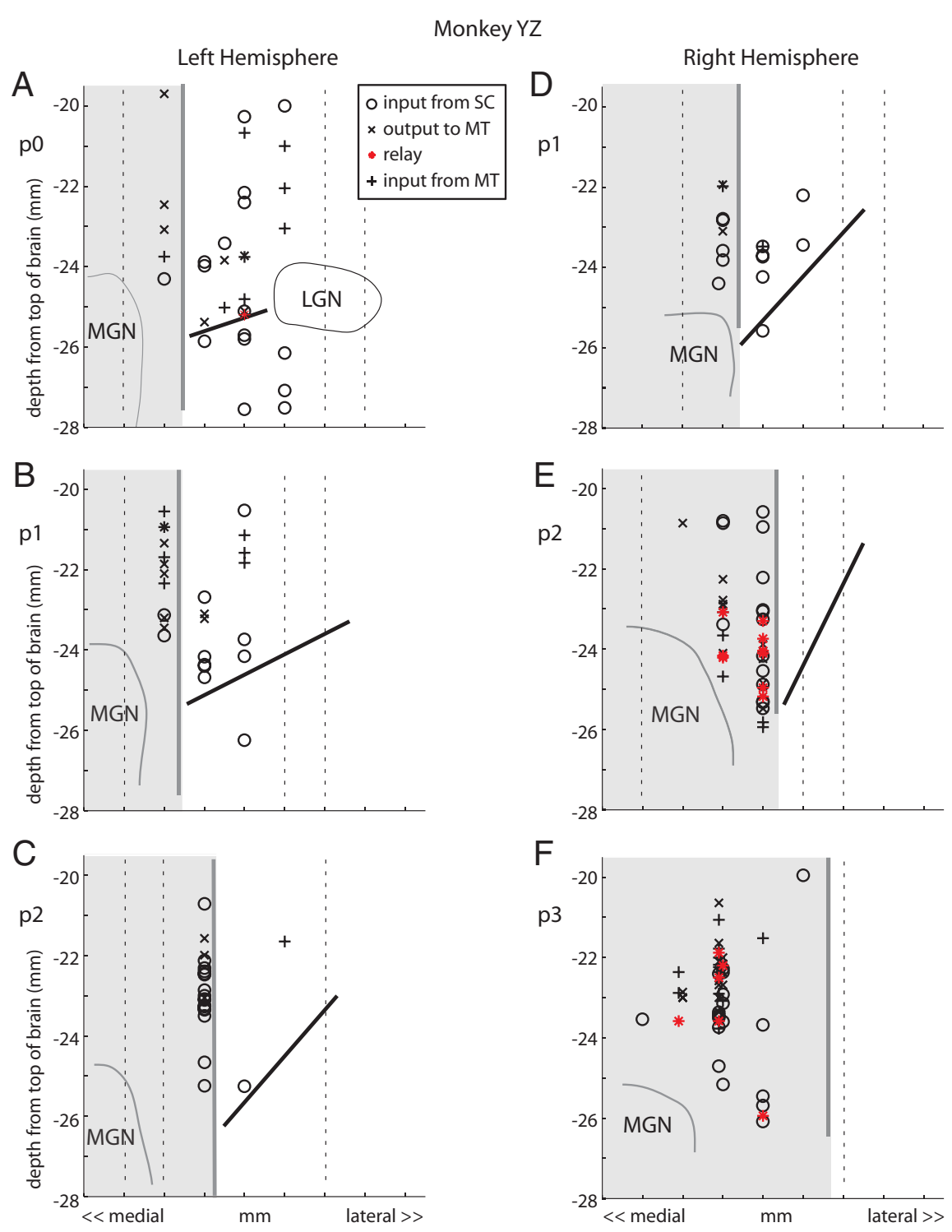

Figure 4. Location of pulvinar neurons with connections to SC and MT identified using microstimulation. Coronal planes are identical to those in Figure 3 (monkey YZ). Symbols indicate the four types of connections: input from SC, output to MT, relay neurons with both SC input and MT output, and input from MT (see legend). Landmarks for each panel are from Figure 3: MGN, LGN, representation of the horizontal meridian, and estimated border between retinotopic and nonretinotopic zones. Data obtained using slightly curved recording electrodes are shown with a small medial offset (right hemisphere, $\boldsymbol{F}$ only). Dotted lines indicate sites at which recording was done but no connected neurons were encountered. Conventions as in Figure 3.

tent with the P3 field identified anatomically by Ungerleider and colleagues, which lacked obvious topographical organization (Ungerleider et al., 1984; Adams et al., 2000; Shipp, 2001; but see also Standage and Benevento, 1983). In the right hemisphere, which we mapped subsequently, the coronal sections begin just posterior to LGN (Fig. 3D-F, section P1). Here we also found a transition from lower to upper visual fields in the lateral part of the pulvinar, as well as a more orderly progression of receptive field eccentricity in this retinotopic zone. We again observed a nonretinotopic zone more medially. In both hemispheres, we found that the nonretinotopic zone represented a larger portion of the mapped area as we moved posteriorly from the LGN, consistent with previous anatomical topography (Adams et al., 2000). These maps demonstrate the presence of retinotopic and nonretinotopic zones and provide an important foundation for localization of the pulvinar neurons that belong to the ascending SC-MT pathway.

\section{Identification of pulvinar neurons connected to SC and MT}

Our next objective was to identify pulvinar cells with connections to SC and MT and determine their location within the retinotopic and nonretinotopic maps. Figure 4 shows the location of connected pulvinar neurons in both hemispheres of monkey YZ, for the same coronal sections of Figure 3. This figure provides a general overview of the types of connected cells we encountered: those with input from SC, output to MT, and input from MT. Most notably, these maps illustrate that SC-MT relay neurons (shown in red) are among the identified cell types in the pulvinar. In the first hemisphere we mapped (Fig. $4 A-C$, left), we identified only one relay neuron, which was located near the LGN. In the right hemisphere (Fig. $4 D-F$ ), we identified more relay neurons, likely due to an improved alignment of visual representations in SC and MT stimulation sites. Relay neurons in this hemisphere were clustered medially near the MGN, $\sim 2 \mathrm{~mm}$ behind the LGN. We did not identify relay neurons just adjacent to the LGN, possibly because the area around LGN was sampled less frequently in this hemisphere. This figure points to two general observations. First, in both hemispheres, the pulvinar neurons with connections to SC and MT are in both the retinotopic and nonretinotopic zones. Second, data from the right hemisphere, in which relay neurons were found, indicate that relay neurons are more concentrated in the nonretinotopic zone.

\section{Location of SC-MT relay neurons with respect to known subdivisions of inferior pulvinar}

Previous work on the putative ascending pathway from SC to pulvinar to MT has centered on the subdivision of the pulvinar known to project densely to MT. Several lines of evidence have suggested that this subdivision, called PIm, is the relay zone linking SC to MT (Cusick et al., 1993; Gutierrez et al., 1995). Recent anatomical work, however, has cast doubt on this hypothesis (Stepniewska et al., 2000). Therefore it was of great interest to know whether the identified relay neurons were located within PIm. To find out, we made electrolytic marks at two sites at which we recorded from relay neurons, and subsequently processed the tissue for the calcium-binding protein calbindin, which distinguishes PIm and other subdivisions of the visual pulvinar. We describe the histological results in four stages. First, we show the calbindin-stained section through the coronal plane in which we marked relay neurons, $\sim 2 \mathrm{~mm}$ posterior to LGN (Fig. $5 A$ ). PIm stands out on the calbindin stain because it stains very lightly whereas its neighboring subdivisions stain darkly, and accordingly is called the "calbindin hole" (Cusick et al., 1993; Gutierrez et al., 1995; Stepniewska and Kaas, 1997; Adams et al., 2000). Second, we superimpose the outline of these calbindin-defined 

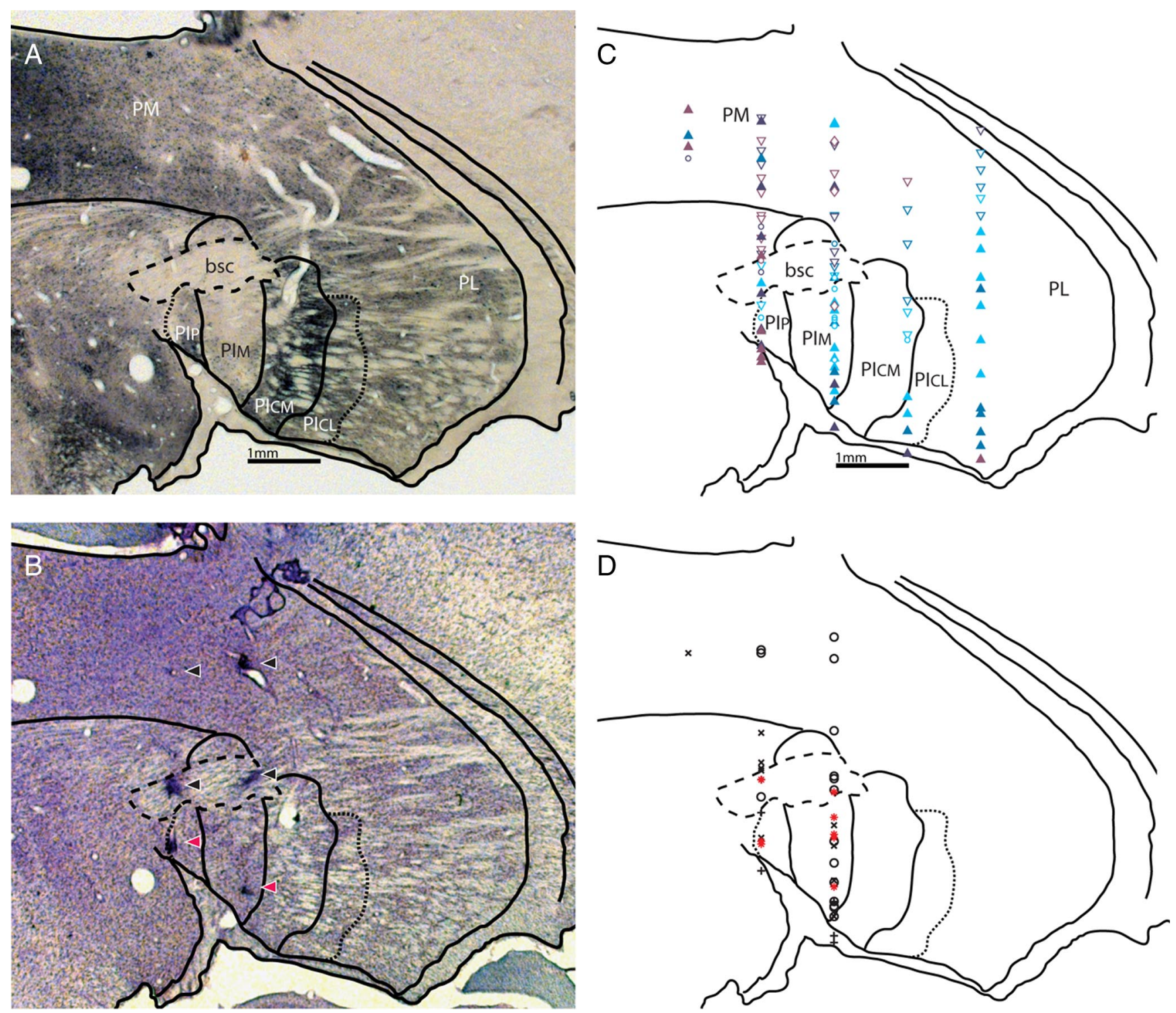

Figure 5. Histological sections from the right hemisphere of monkey YZ show that relay neurons are located in the known MT-projection zone as well as an adjacent subdivision of inferior pulvinar. $A$, Section stained for calbindin reveals known subdivisions within the inferior pulvinar for the coronal plane in which relay neurons were identified. Solid lines indicate calbindin-identified subdivisions and other landmarks; the dashed line indicates the brachium of the SC (bsc), a fiber passage in which pulvinar neurons are interdigitated (Adams et al., 2000). Pulvinar labels follow the nomenclature of Stepniewska and Kaas (1997): Plp, inferior pulvinar posterior subdivision; PIm, medial subdivision; PIcm, central-medial subdivision; PIcl, central-lateral subdivision. Dotted lines for Plp indicate less certainty regarding its medial and dorsal border due to damage at the site of the electrolytic mark; it may extend more medially and above the brachium. The lateral border of PIcl was also less certain. The lightly stained region (calbindin hole) corresponds to the area known to project most densely to MT (PIm). $\boldsymbol{B}$, The adjacent Nissl section shows the pattern of marks made by passing current through the recording microelectrode. Arrowheads identify marks on two penetrations including two sites at which relay neurons (red arrowheads) were recorded. Marks were used to align recording maps for $\boldsymbol{C}$ and $\boldsymbol{D}$. C, Receptive field map from Figure $3 E$ is superimposed on the outline of calbindin-defined subdivisions. $\boldsymbol{D}$, The map of pulvinar neurons connected to $S \boldsymbol{C}$ and $M T$, from Figure $4 E$, is superimposed on the calbindin outline. Map conventions for $C$ and $\boldsymbol{D}$ are from Figures 3 and 4 , respectively. Relay neurons (red) were recorded both in PIm and in the more medial subdivision Plp.

subdivisions on the adjacent section stained for Nissl (Fig. 5B). The Nissl section shows the electrolytic marks at the site of the two identified relay neurons (red arrows), which were recorded in adjacent penetrations in the same coronal plane, separated by $1 \mathrm{~mm}$ mediolateral. Also shown are the more superficial locations where additional marks created a recognizable pattern for each penetration (black arrows). Together these marks served as anchors to relate our recording to the calbindindefined subdivisions. Third, we show the relationship between the receptive field maps and the calbindin subdivisions (Fig. 5C). Superimposed on the calbindin-defined outline is the map of receptive fields for this section, from Figure $3 E$. The areas defined as PIm and its more medial subdivision, PIp, fall within the medial nonretinotopic zone, consistent with earlier findings (Ungerleider et al., 1984; Adams et al., 2000; Shipp, 2001), although it remains possible that these medial subdivisions have greater topography than typically detected (e.g., Standage and Benevento, 1983). The more lateral subdivisions of the inferior pulvinar correspond to our retinotopic zone, again consistent with anatomical findings with the possible exception of the PIcm subdivision, which we had predicted to be largely nonretinotopic but did not sample sufficiently (Adams et al., 2000). Finally, in Figure $5 D$, we show the relationship of greatest interest: where are the connected neurons, particularly the relay neurons, with re- 
spect to the calbindin-defined subdivisions? Here we have overlaid the maps of the connected cells from Figure $4 E$, which clearly show that relay neurons are indeed found within the PIm subdivision. They are not limited to PIm, however. They are also found in the more medial subdivision, PIp.

\section{Generality of the localization of SC-MT relay neurons and connected cells}

The detailed mapping in monkey YZ allowed us to establish the location of relay neurons and other SC- and MT-connected cells with respect to physiological receptive field maps and immunohistochemical subdivisions of the pulvinar. We subsequently recorded in an additional three hemispheres. During this stage of the experiments, we took advantage of the landmarks from monkey YZ and conducted more targeted recording in lieu of extensive and detailed mapping. Figure 6 shows the coronal maps of connected cells from these three hemispheres, which were combined using the LGN and MGN as landmarks. We identified relay neurons in each of these three hemispheres. Once again, the most prominent cluster was near the MGN and posterior to the LGN, but we also identified a handful of relays immediately adjacent to the LGN.

We summarized the location of all pulvinar neurons with SC and MT connections, including relays, by creating a topdown map of the pulvinar (Fig. 7). This map combined data from all three monkeys (five hemispheres). We chose the right hemisphere from monkey YZ as a template, and then used the posterior LGN as a landmark to convert the medial-lateral and anterior-posterior coordinates from the other four hemispheres to this common template. The composite map for relay neurons (Fig. 7) indicates that they were in greatest concentration near the MGN at the posterior and medial edge of the visual pulvinar (lower part of the map), $\sim 2-3 \mathrm{~mm}$ posterior and 1-2 mm medial to the posterior aspect of LGN. A smaller cluster was found adjacent to and just below the LGN (upper right part of the map).

We then constructed composite maps for the locations of all neurons connected to SC and MT (Fig. 8). We can make three observations from these maps. First, they establish the general borders of the pulvinar region containing cells connected to SC and MT. These connected cells were found as far anterior as the posterior aspect of the LGN, and although depth is not represented here, they were also found in small numbers directly beneath this part of LGN. The connected cells were located up to 3 $\mathrm{mm}$ posterior to the LGN. In our recordings, we found that this corresponded to the posterior border of the visual pulvinar; neurons encountered more posteriorly were nonvisual in nature and difficult to characterize. In the medial-lateral dimension, the majority of connected neurons were located in a $2-3 \mathrm{~mm}$ zone demarcated by the MGN and LGN. The second observation is that these maps indicate considerable overlap in the location of neurons with SC input and MT output. Despite this overlap, we found a small but significant difference in the mediolateral location of SC-connected cells compared with MT-connected cells. Overall, neurons with either MT output or MT input were slightly more medial than those with SC input (median difference of $0.5 \mathrm{~mm} ; p<0.001$ and $p<0.0167$, significant after Bonferroni correction, excluding relay neurons). This small difference was also significant in the three individual hemispheres with sufficient sample sizes, and may reflect the predominance of MT connectivity in the medial aspects of the inferior pulvinar (Lin and Kaas, 1980; Ungerleider et al., 1984; Cusick et al., 1993; Adams et al., 2000). Third, the maps of MT output neurons and
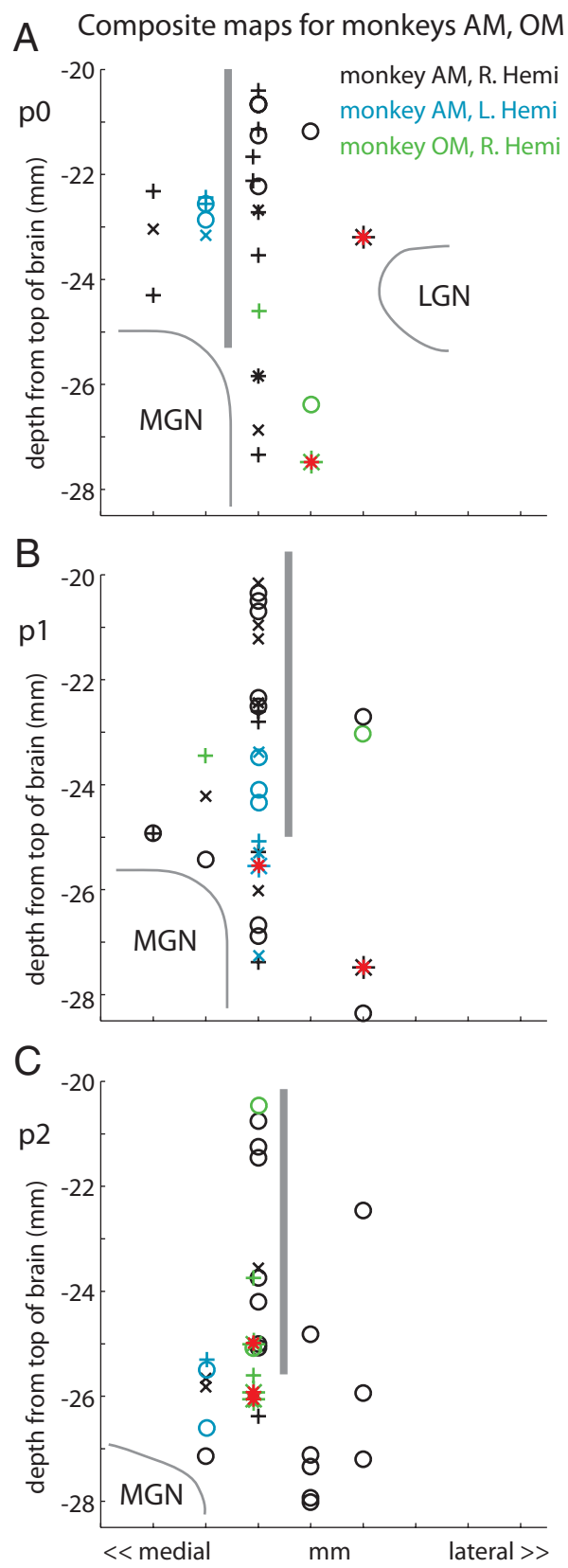

Figure 6. Coronal maps show the location of identified pulvinar neurons sampled less densely from three other hemispheres. Each hemisphere is represented with a different color (see legend). The primary landmarks (MGN, LGN, and estimated border between retinotopic and nonretinotopic zones) are from the right hemisphere of monkey AM; data from the other two hemispheres were brought into register on the basis of MGN and LGN locations. Conventions as in Figure 4. R, Right; L, left; Hemi, hemisphere.

MT input neurons are nearly identical, in keeping with a reciprocity of connections between pulvinar and cortex.

\section{The interaction between pulvinar and cortex: output to and input from MT}

The central goal of our investigation was to identify the ascending path from SC to pulvinar to MT, but stimulation in MT also permitted the identification of pulvinar neurons that received descending input from MT. As noted in the previous section, the location of neurons with input from MT overlapped considerably with those that projected to MT. We were unable to detect, how- 


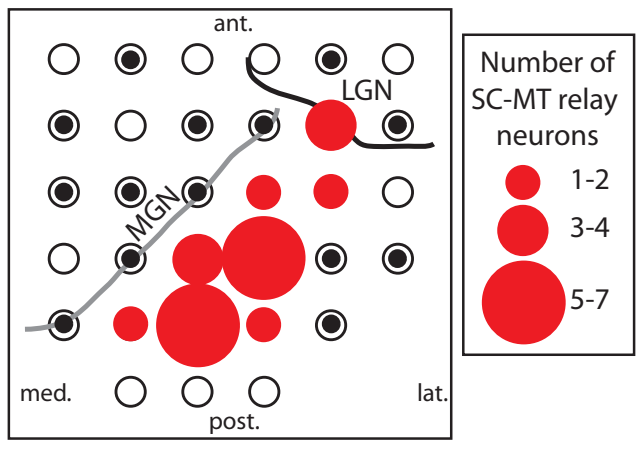

Figure 7. Top-down view of a composite grid map of the pulvinar shows the anteriorposterior and medial-lateral location of relay neurons. Data from all three monkeys ( 5 hemispheres) were brought into a common coordinate frame and superimposed onto the chamber map from the right hemisphere of monkey YZ. Lines represent the estimated posterior/medial border of LGN (black) and the lateral border of MGN (gray). Filled red circles denote the location of relay neurons; their diameter reflects the number at each site. Open circles indicate grid locations at which recording was conducted, and small black circles denote sites of nonrelay neurons that were connected to either SC or MT. ant., Anterior; post., posterior; lat., lateral; med., medial.

ever, any single neurons that clearly received input from MT and also projected to the same part of MT. In other words, when we stimulated at one of the MT sites, the same stimulation did not evoke both an orthodromic and an antidromic spike from the recorded pulvinar neuron. It is possible that higher currents may have revealed an orthodromic spike in addition to a detected antidromic spike, or vice versa. There are at least two possible explanations for this absence of obvious reciprocal connectivity in single neurons. One is that the pulvinar does contain single neurons that both receive from and project to MT but we failed to identify them. Another more interesting explanation is that the pulvinar neurons that receive input from MT are genuinely distinct from those that send output to MT. The possibility of separate populations is in keeping with known laminar distinctions within cortex. Pulvinar afferents to visual cortex arrive predominantly in layers 1 and 3, while most projections from cortex to the pulvinar originate in layer 5 (Ogren and Hendrickson, 1976; Trojanowski and Jacobson, 1976, 1977; Lund et al., 1981). More generally, the presence of distinct populations would be consistent with the hypothesis that the pulvinar acts as a higherorder relay, serving as a site for corticocortical interaction (Guillery and Sherman, 2002; Shipp, 2003). A tenet of this hypothesis is that single pulvinar neurons would project to and receive input from different cortical representations. For example, a neuron that projects to the part of MT representing the fovea would not receive input back from this same area but at minimum would receive input from a part of MT with a different spatial representation, if not from another cortical area altogether.

\section{Timing in the SC-pulvinar-MT pathway}

The use of microstimulation to identify pulvinar cells connected to SC and MT also gives us information about the timing of interactions between these structures. We measured activation latency, the time it takes to evoke a spike in the pulvinar after SC or MT stimulation. For cases of antidromic activation (pulvinar neurons projecting to MT), this measure closely reflects the time for transmitting an action potential along the axons. For cases of orthodromic activation (pulvinar neurons receiving input from either SC or MT), this latency includes transmission time as well
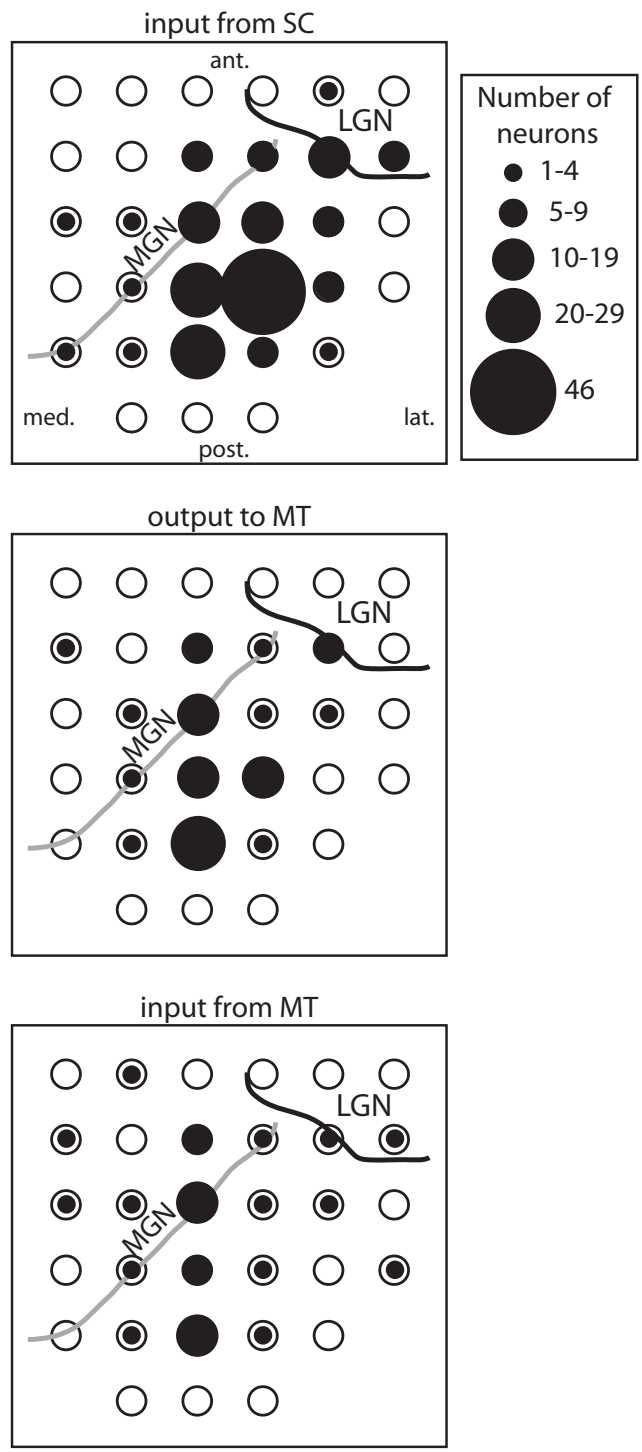

Figure 8. Composite map of all neurons with connections to SC or MT, combined from all five hemispheres. Each map shows the anterior-posterior and medial-lateral location of pulvinar neurons with connections to either SC or MT. $\boldsymbol{A}$, Input from SC. $\boldsymbol{B}$, Output to MT. $\boldsymbol{C}$, Input from MT. Relay neurons are included in the counts for input from SC and output to MT. Conventions as in Figure 7. ant., Anterior; post., posterior; lat., lateral; med., medial.

as the synaptic delay. We found a range of activation latencies for all three of the major connections (input from SC, output to MT, and input from MT) (Fig. 9). The activation latencies of relay neurons differed slightly from the overall population of SC input and MT output neurons, and accordingly are shown separately in red. Overall, median latencies were $3 \mathrm{~ms}$ for orthodromic pulvinar activation from the SC, i.e., it took $3 \mathrm{~ms}$ for an action potential to travel from SC and synaptically drive the pulvinar (Fig. $9 A$ ). This activation latency from SC was slightly shorter for relay neurons but not significantly so. Median antidromic latencies from MT back to pulvinar were $2.2 \mathrm{~ms}$ overall (Fig. $9 B$ ). The relay neurons had a significantly shorter median antidromic latency than did the neurons for which only the MT output was identified. Orthodromic activation from MT (Fig. 9C) was comparable to that from SC. Analysis of the entire sample, including relay neurons, indicated that antidromic latencies for the MT output neurons were significantly shorter than either the SC input latencies $(p<0.0001)$ or the MT input latencies $(p<0.001$, Wil- 
A

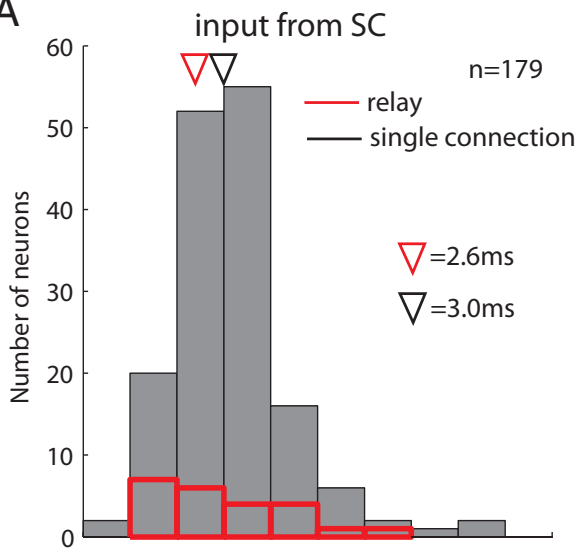

B

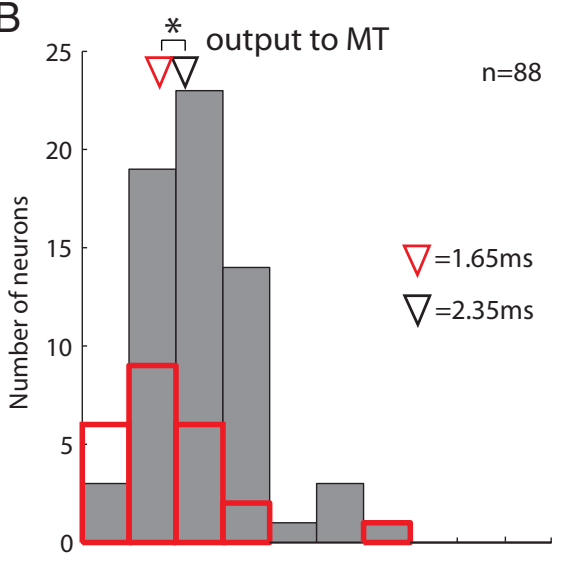

C

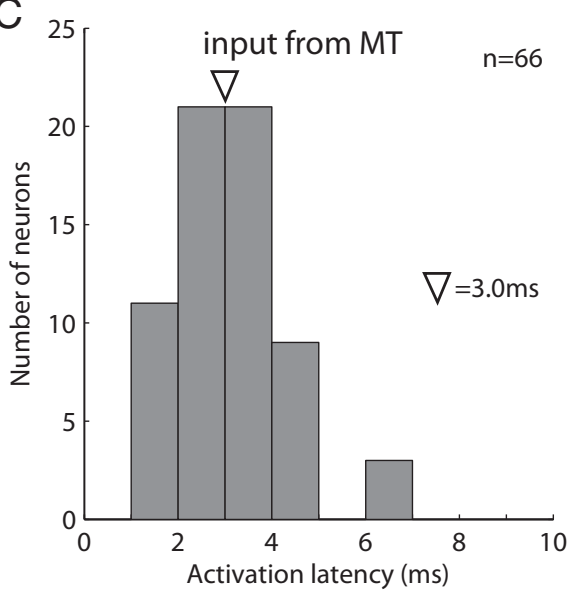

Figure 9. $\quad A-C$, Activation latencies of pulvinar neurons with input from $S C(A)$, output to MT $(\boldsymbol{B})$, and input from MT (C). Distribution of relay neurons are shown in red in $\boldsymbol{A}$ and $\boldsymbol{B}$. In each panel, the $x$-axis shows the time in ms between the start of stimulation of SC or MT and the evoked spike in the pulvinar neuron, and the $y$-axis shows the number of neurons. Histograms were created using $1 \mathrm{~ms}$ bins. Arrows indicate median values, listed on the right. Numbers for the $\mathrm{SC}$ input population differ slightly from the total of identified neurons in this category because precise time measurements were not obtained for a small number of cells. Asterisk in $\boldsymbol{B}$ indicates a significant difference between antidromic MT latencies for the relay neurons compared with those with only identified MT output ( $p<0.05$, Wilcoxon rank-sum).

coxon rank-sum test, significant after Bonferroni correction). The shorter latencies for antidromic activation from MT are consistent with backfiring the neuron rather than driving it synaptically, i.e., these latencies do not include the synaptic delay inherent to the measure of orthodromic latencies. Without this synaptic delay (typically $0.5-1 \mathrm{~ms}$ ), it is possible that the pulv- inar neurons with MT input and MT output would have similar activation latencies and thus comparable conduction velocities. A true comparison of timing for the pulvinar-MT and MT-pulvinar projections would require a direct antidromic measure of MT-pulvinar transmission (by recording in MT during pulvinar stimulation). Our data cannot address this possibility, but point toward additional information that could shed light on the interactions that arise between the pulvinar and subtypes of cortical neurons associated with specific layers (Rockland, 1996; Crick and Koch, 1998; Rockland et al., 1999; Van Horn and Sherman, 2004).

How rapidly can signals ascend from SC to pulvinar to MT? We can approximate the total travel time in relay neurons by summation of the orthodromic latencies from SC stimulation and the antidromic latencies from MT stimulation. This estimate, while a simplification, has been shown to be a good predictor of actual measured travel times in another ascending pathway, from SC to mediodorsal thalamus (MD) to frontal cortex (Sommer and Wurtz, 2004). In the pulvinar path, we found that the shortest predicted travel time from SC to pulvinar to MT for the sample of relay neurons was $2.3 \mathrm{~ms}$ but the median value was $5 \mathrm{~ms}$, slower than the median travel time of $2.4 \mathrm{~ms}$ observed in the ascending path through MD (Sommer and Wurtz, 2004). Transmission times through the pulvinar are nonetheless reasonably fast, and considerably faster than those predicted by earlier estimates of the connections from SC to pulvinar (Marrocco et al., 1981).

\section{Discussion}

An ongoing controversy in understanding the function of the visual pulvinar has centered on its possible role as a relay from subcortical structures to visual cortex. Here we used the techniques of antidromic and orthodromic stimulation to identify a pathway from the midbrain (SC) to the cerebral cortex (MT) though the pulvinar. Three key observations emerged. First, we identified many neurons in the visual pulvinar that received input from SC or sent information to MT; we also identified a largely distinct population of neurons that received input from MT. Second, and significantly, we found that a subset of these neurons were relay neurons that both received SC input and projected to MT. The identification of these relay neurons establishes beyond any doubt a functional path from SC to MT through pulvinar. Third, we localized a subset of relay neurons to well established subdivisions of the pulvinar. Relay neurons were located in area PIm of the inferior pulvinar, a subdivision known to have dense connectivity with area MT. Notably, however, we also localized relay neurons to the medial-most subdivision, PIp. This pattern indicates that the pathway through the pulvinar is not confined to one anatomical subdivision. The identification of this pathway has important implications for understanding how the pulvinar is organized and how this organization supports the transmission of signals to cortex.

Relay neurons and a new landscape of pulvinar organization How does the location of relay neurons inform the present understanding of the anatomical organization of the visual pulvinar? Our data, in conjunction with anatomical findings, indicate the presence of at least two relay zones in the pulvinar that link SC and MT. For simplicity, we refer to these as an MGN-adjacent zone and an LGN-adjacent zone. The MGN-adjacent zone, located immediately lateral to MGN and several millimeters posterior and medial to LGN, is where we identified the majority of relay neurons. Importantly, subsequent histology confirmed that 
some of the relays in this zone resided in two subdivisions defined by immunostaining, PIm and PIp. PIm has been at the center of the debate regarding the possible pulvinar relay from SC to MT. Previous anatomical studies contended that PIm projected to MT but received no evident input from SC (Stepniewska et al., 2000). Our results show that there are not only relay neurons in PIm but also a number of neurons with input from SC. In this regard our findings are consistent with previous observations that suggested PIm as a relay. PIm has a signature pattern of immunohistochemical staining found in other known relay nuclei such as the LGN and MGN_namely, it stains darkly for cytochrome oxidase and parvalbumin but lightly for calbindin (Cusick et al., 1993; Gutierrez et al., 1995). This observation, coupled with anatomical data showing dense projections to area MT (Lin and Kaas, 1980; Cusick et al., 1993), led to the hypothesis that PIm was a relay subdivision. Our identification of relay neurons in PIm bears out these predictions and demonstrates that PIm participates in relaying information from SC to MT.

Importantly, relay neurons in the MGN-adjacent zone were not limited to PIm but were also found in a more medial subdivision of the inferior pulvinar called PIp. The presence of relay neurons here is consistent with the anatomical data of Adams et al. (2000). These investigators found that in addition to the prominent projection to MT from PIm, projections to MT also originated in two flanking subdivisions, PIp and PIcm. The major projections of PIp and PIcm were directed to area V4 (Adams et al., 2000), but their connectivity emphasizes a role in multiple visual functions. These subdivisions contain neurons that project to other motion areas outside MT (Kaas and Lyon, 2007) and they are a major target of input from the superficial SC (Lin and Kaas, 1979; Stepniewska et al., 2000). The localization of SC-MT relay neurons to PIp reinforces the idea that the architectonic subdivisions of the pulvinar are not devoted exclusively to a single function, and instead may support interaction between specialized cortical visual areas (Guillery and Sherman, 2002; Shipp, 2003).

In addition to the MGN-adjacent zone for relay neurons, our findings converge with recent anatomical results to indicate the presence of a second, LGN-adjacent zone. The relay neurons we found near and below the posterior pole of LGN are likely within a region identified in a transneuronal tracer study by Lyon and colleagues (2005). These investigators injected a rabies virus into MT to reveal its second-order afferents (Kelly and Strick, 2000) and demonstrated that SC projects disynaptically to MT. They also found labeled cells in the pulvinar that could constitute the relay, clustered near LGN in a region that probably corresponds to a subdivision called the "lateral shell" (Cusick et al., 1993; Gutierrez et al., 1995). Our peri-LGN relay neurons are consistent with this lateral projection. Together, the previous anatomical observations and our present electroanatomical findings constitute evidence for the existence of at least two relay zones that ascend from SC to MT through the pulvinar.

We have laid out evidence for two SC-MT relay zones, but do not mean to suggest that they are the only sites; clearly, identified relay neurons were not tightly restricted to two circumscribed, noncontiguous zones. An intriguing speculation is that some of the relay neurons correspond to the "giant" neurons identified by Jones (2007) and recently shown to be pulvinocortical relays (Imura and Rockland, 2007). These giant neurons are distinguished by their immunoreactivity and appear to be very sparsely distributed in the inferior pulvinar, especially in its lateral aspects. Some of our identified relay neurons, particularly those outside the MGN-adjacent cluster, may belong to this population.

\section{Limitations}

The combination of orthodromic and antidromic stimulation that we used to identify SC-MT relay neurons provides strong evidence for a pulvinar pathway that connects SC to MT. The stimulation technique also has two notable limitations. The first limitation is the small number of identified relay neurons, 24 compared with 223 "half-relays" that either received input from SC or sent output to MT but not both. Several factors may contribute to this ratio. One is that the relay neurons may be genuinely sparse. This possibility is consistent with the absence of strong anatomical evidence for the relay when investigated by Stepniewska et al. (2000) and may also be consistent with the observation of sparse relay neuron candidates (Imura and Rockland, 2007). Another factor stems from the limitations of the antidromic/orthodromic stimulation technique: it provides strong evidence when successful activation indicates a connection but no evidence when it does not. Failure to activate a neuron can have a number of causes. A major one, however, must be the lack of spatial alignment between the visual field representations at the two stimulation sites and at the recording electrode. Consequently, the number of relay neurons we observe is probably the lower limit.

The second limitation concerns the possibility that stimulation in the superficial layers of SC may activate not only neurons that project from SC to the pulvinar, but also cortical axons that descend to SC, likely from layer 5 of visual cortex. Axonal reconstructions in the squirrel monkey have shown that a fraction of V1 neurons with projections to SC also have a collateral branch within the pulvinar (Rockland, 1998). SC stimulation could generate an antidromic spike in these descending cortical axons and produce an orthodromic input to the pulvinar via the collateral branch. Such collateral activation has been observed in cat for corticopontine axons that bifurcate below the SC and send a long collateral back to SC (Baker et al., 1983). For the collateral to the pulvinar, we have no information about the strength of the collateral synapse or the frequency with which an antidromic spike could activate the pulvinar (the safety factor). When we consider the visual responses of pulvinar neurons activated by SC stimulation, however, the ascending SC projection appears to be the stronger determining input. The SC-activated pulvinar neurons have receptive fields driven by spot stimuli (Berman and Wurtz, 2008) (R. A. Berman and R. H. Wurtz, unpublished data) similar to those of the SC (Goldberg and Wurtz, 1972), rather than the orientation-selective and smaller receptive fields of layer $5 \mathrm{~V} 1$ neurons (Schiller et al., 1976; Gur et al., 2005). The simplest explanation of the data is that the majority of SC-activated pulvinar neurons are stimulated via the ascending projection from SC to pulvinar, but even so a fraction may also (or only) receive cortical input. A definitive resolution depends on the challenging experiment of severing the descending cortical axons to SC and verifying that the activation of pulvinar from SC remains.

\section{Ascending pathways from brainstem to cortex}

The pulvinar path from SC to MT might be considered a posterior complement to the pathway that ascends from SC to frontal cortex via MD thalamus. The pulvinar path originates in the superficial, visual layers of SC and projects to area MT in parietooccipital cortex, whereas the MD path originates in the intermediate, saccade-related layers of SC and projects to the frontal eye field. In this regard, the two pathways share key features-each originates in a specific part of the SC and projects to a specific cortical subregion, linking a subcortical spatial map to a cortical one. Yet their distributions in the intervening thalamic nucleus 
appear to differ: relay neurons were tightly clustered in a small zone in MD (Sommer and Wurtz, 2004) but were less concentrated in the pulvinar. This may reveal a fundamental organizational feature of the pulvinar in that the pathways through it are not restricted to a single zone. Nevertheless, the fact that we have identified two likely relay zones in the pulvinar path-particularly the predominant cluster near MGN_now makes it possible to answer causal questions about the pulvinar pathway as was done in the MD path from SC to frontal cortex (Sommer and Wurtz, 2002, 2006). Furthermore, the physiological identification of this pathway permits the characterization of the neuronal signals it conveys. Progress in these endeavors promises not only to elucidate the complexities of the pulvinar but also to inform the understanding of how subcortical and cortical mechanisms interact to produce visual perception.

Note added in proof. At the time the current article was going to press, the cited anatomical work of Lyon and colleagues (2005) was published in an article that describes more fully the disynaptic SC-MT pathway (Lyon et al., 2010).

\section{References}

Adams MM, Hof PR, Gattass R, Webster MJ, Ungerleider LG (2000) Visual cortical projections and chemoarchitecture of macaque monkey pulvinar. J Comp Neurol 419:377-393.

Baizer JS, Whitney JF, Bender DB (1991) Bilateral projections from the parabigeminal nucleus to the superior colliculus in monkey. Exp Brain Res 86:467-470.

Baker J, Gibson A, Mower G, Robinson F, Glickstein M (1983) Cat visual corticopontine cells project to the superior colliculus. Brain Res 265:227-232.

Bender DB (1981) Retinotopic organization of macaque pulvinar. J Neurophysiol 46:672-693.

Benevento LA, Fallon JH (1975) The ascending projections of the superior colliculus in the rhesus monkey (Macaca mulatta). J Comp Neurol 160:339-361.

Benevento LA, Standage GP (1983) The organization of projections of the retinorecipient and nonretinorecipient nuclei of the pretectal complex and layers of the superior colliculus to the lateral pulvinar and medial pulvinar in the macaque monkey. J Comp Neurol 217:307-336.

Berman RA, Wurtz RH (2008) Exploring the pulvinar path to visual cortex. Prog Brain Res 171:467-473.

Celio MR (1990) Calbindin D-28k and parvalbumin in the rat nervous system. Neuroscience 35:375-475.

Crick F, Koch C (1998) Constraints on cortical and thalamic projections: the no-strong-loops hypothesis. Nature 391:245-250.

Crist CF, Yamasaki DS, Komatsu H, Wurtz RH (1988) A grid system and a microsyringe for single cell recording. J Neurosci Methods 26:117-122.

Cusick CG, Scripter JL, Darensbourg JG, Weber JT (1993) Chemoarchitectonic subdivisions of the visual pulvinar in monkeys and their connectional relations with the middle temporal and rostral dorsolateral visual areas, MT and DLr. J Comp Neurol 336:1-30.

Diamond IT, Hall WC (1969) Evolution of neocortex. Science 164:251-262.

Fries W (1984) Cortical projections to the superior colliculus in the macaque monkey: a retrograde study using horseradish peroxidase. J Comp Neurol 230:55-76.

Goldberg ME, Wurtz RH (1972) Activity of superior colliculus in behaving monkey. I. Visual receptive fields of single neurons. J Neurophysiol 35: 542-559.

Grieve KL, Acuña C, Cudeiro J (2000) The primate pulvinar nuclei: vision and action. Trends Neurosci 23:35-39.

Guillery RW, Sherman SM (2002) Thalamic relay functions and their role in corticocortical communication: generalizations from the visual system. Neuron 33:163-175.

Gur M, Kagan I, Snodderly DM (2005) Orientation and direction selectivity of neurons in V1 of alert monkeys: functional relationships and laminar distributions. Cereb Cortex 15:1207-1221.

Gutierrez C, Yaun A, Cusick CG (1995) Neurochemical subdivisions of the inferior pulvinar in macaque monkeys. J Comp Neurol 363:545-562.

Harting JK, Huerta MF, Frankfurter AJ, Strominger NL, Royce GJ (1980)
Ascending pathways from the monkey superior colliculus: an autoradiographic analysis. J Comp Neurol 192:853-882.

Hays AV, Richmond BJ, Optican LM (1982) A UNIX-based multiple process system for real-time data acquisition and control. WESCON Conf Proc 2:1-10.

Imura K, Rockland KS (2007) Giant neurons in the macaque pulvinar: a distinct relay subpopulation. Front Neuroanat 1:2.

Jones EG (2007) The thalamus, Ed 2. Cambridge, UK: Cambridge UP.

Kaas JH, Lyon DC (2007) Pulvinar contributions to the dorsal and ventral streams of visual processing in primates. Brain Res Rev 55:285-296.

Kelly RM, Strick PL (2000) Rabies as a transneuronal tracer of circuits in the central nervous system. J Neurosci Methods 103:63-71.

Lemon R (1984) Methods for neuronal recording in conscious animals. In: IBRO handbook series: methods in the neurosciences. Vol 4, pp 95-102. New York: Wiley.

Lin CS, Kaas JH (1979) The inferior pulvinar complex in owl monkeys: architectonic subdivisions and patterns of input from the superior colliculus and subdivisions of visual cortex. J Comp Neurol 187:655-678.

Lin CS, Kaas JH (1980) Projections from the medial nucleus of the inferior pulvinar complex to the middle temporal area of the visual cortex. Neuroscience 5:2219-2228.

Lund JS, Hendrickson AE, Ogren MP, Tobin EA (1981) Anatomical organization of primate visual cortex area VII. J Comp Neurol 202:19-45.

Lyon DC, Nassi JJ, Callaway EM (2005) Primate dorsal stream visual areas receive disynaptic inputs from the superior colliculus. Soc Neurosci Abstr 31:137.136.

Lyon DC, Nassi JJ, Callaway EM (2010) A disynaptic relay from superior colliculus to dorsal stream visual cortex in macaque monkey. Neuron 65:270-279.

Malpeli JG, Baker FH (1975) The representation of the visual field in the lateral geniculate nucleus of Macaca mulatta. J Comp Neurol 161: 569-594.

Marrocco RT, McClurkin JW, Young RA (1981) Spatial properties of superior colliculus cells projecting to the inferior pulvinar and parabigeminal nucleus of the monkey. Brain Res 222:150-154.

Maunsell JH, Newsome WT (1987) Visual processing in monkey extrastriate cortex. Annu Rev Neurosci 10:363-401.

Movshon JA, Newsome WT (1996) Visual response properties of striate cortical neurons projecting to area MT in macaque monkeys. J Neurosci 16:7733-7741.

Ogren M, Hendrickson A (1976) Pathways between striate cortex and subcortical regions in Macaca mulatta and Saimiri sciureus: evidence for a reciprocal pulvinar connection. Exp Neurol 53:780-800.

Ottes FP, Van Gisbergen JA, Eggermont JJ (1986) Visuomotor fields of the superior colliculus: a quantitative model. Vision Res 26:857-873.

Petersen SE, Robinson DL, Keys W (1985) Pulvinar nuclei of the behaving rhesus monkey: visual responses and their modulation. J Neurophysiol 54:867-886.

Robinson DL (1993) Functional contributions of the primate pulvinar. Prog Brain Res 95:371-380.

Rockland KS (1996) Two types of corticopulvinar terminations: round (type 2) and elongate (type 1). J Comp Neurol 368:57-87.

Rockland KS (1998) Convergence and branching patterns of round, type 2 corticopulvinar axons. J Comp Neurol 390:515-536.

Rockland KS, Andresen J, Cowie RJ, Robinson DL (1999) Single axon analysis of pulvinocortical connections to several visual areas in the macaque. J Comp Neurol 406:221-250.

Saleem KS, Logothetis NK (2007) A combined MRI and histology atlas of the rhesus monkey brain in stereotaxic coordinates. London: Elsevier.

Sanders MD, Warrington EK, Marshall J, Wieskrantz L (1974) "Blindsight": vision in a field defect. Lancet 1:707-708.

Schiller PH, Malpeli JG (1977) Properties and tectal projections of monkey retinal ganglion cells. J Neurophysiol 40:428-445.

Schiller PH, Finlay BL, Volman SF (1976) Quantitative studies of single-cell properties in monkey striate cortex. I. Spatiotemporal organization of receptive fields. J Neurophysiol 39:1288-1319.

Shipp S (2001) Corticopulvinar connections of areas V5, V4, and V3 in the macaque monkey: a dual model of retinal and cortical topographies. J Comp Neurol 439:469-490.

Shipp S (2003) The functional logic of cortico-pulvinar connections. Philos Trans R Soc Lond B Biol Sci 358:1605-1624.

Sommer MA, Wurtz RH (2000) Composition and topographic organiza- 
tion of signals sent from the frontal eye field to the superior colliculus. J Neurophysiol 83:1979-2001.

Sommer MA, Wurtz RH (2002) A pathway in primate brain for internal monitoring of movements. Science 296:1480-1482.

Sommer MA, Wurtz RH (2004) What the brain stem tells the frontal cortex. I. Oculomotor signals sent from superior colliculus to frontal eye field via mediodorsal thalamus. J Neurophysiol 91:1381-1402.

Sommer MA, Wurtz RH (2006) Influence of the thalamus on spatial visual processing in frontal cortex. Nature 444:374-377.

Standage GP, Benevento LA (1983) The organization of connections between the pulvinar and visual area MT in the macaque monkey. Brain Res 262:288-294.

Stepniewska I, Kaas JH (1997) Architectonic subdivisions of the inferior pulvinar in New World and Old World monkeys. Vis Neurosci 14:10431060.

Stepniewska I, Q1 HX, Kaas JH (2000) Projections of the superior colliculus to subdivisions of the inferior pulvinar in New World and Old World monkeys. Vis Neurosci 17:529-549.
Trojanowski JQ, Jacobson S (1976) Areal and laminar distribution of some pulvinar cortical efferents in rhesus monkey. J Comp Neurol 169:371-392.

Trojanowski JQ, Jacobson S (1977) The morphology and laminar distribution of cortico-pulvinar neurons in the rhesus monkey. Exp Brain Res 28:51-62.

Ungerleider LG, Desimone R, Galkin TW, Mishkin M (1984) Subcortical projections of area MT in the macaque. J Comp Neurol 223:368-386.

Van Essen DC, Maunsell JH, Bixby JL (1981) The middle temporal visual area in the macaque: myeloarchitecture, connections, functional properties and topographic organization. J Comp Neurol 199:293-326.

Van Horn SC, Sherman SM (2004) Differences in projection patterns between large and small corticothalamic terminals. J Comp Neurol 475:406-415.

Zhu JJ, Lo FS (1998) Control of recurrent inhibition of the lateral posteriorpulvinar complex by afferents from the deep layers of the superior colliculus of the rabbit. J Neurophysiol 80:1122-1131. 